\title{
Prevalence and treatment of diabetes mellitus and hypertension among older adults with intellectual disability in comparison with the general population
}

Anna Axmon ${ }^{1,2^{*}}$ (D) Gerd Ahlström² and Peter Höglund ${ }^{3}$

\begin{abstract}
Background: Diabetes mellitus and hypertension are risk factors for cardiovascular disease, which is the most common cause of death in the world. People with intellectual disability (ID) have been reported to have high rates of both these disorders. The aim of this study was to describe and compare prevalence ratios of diabetes mellitus and hypertension between older adults with ID and their age peers in the general population, and to describe and compare treatment patterns in these two groups.
\end{abstract}

Methods: This is a Swedish register-based study, in which we established a cohort of people aged $55+$ years and who had received support for those with ID in $2012(n=7936)$. We also established a same-sized referent cohort from the general population matched by sex and year of birth. Information on diagnoses of diabetes mellitus and hypertension, and prescription of drugs for these disorders, were collected from national registers for the period 2006-2012. The two cohorts were compared using generalized linear models (GLM).

Results: People with ID were 20\% more likely than the general population to have a diagnosis of diabetes mellitus, and $26 \%$ more likely to have prescription of drugs for diabetes mellitus. People in the general population were $81 \%$ more likely to have a diagnosis of hypertension, and $9 \%$ more likely to have a prescription of drugs for hypertension. Among those with diabetes, ID was associated with higher occurrence of prescription of insulin combination drugs and sulfonylureas, but lower occurrence of prescription of dipeptidyl peptidase (DPP) 4-inhibitors and exenatide/liraglutide. Among those with hypertension, ID was associated with higher occurrence of prescription of diuretics, but lower occurrence of prescription of calcium channel blockers and angiotensin II antagonists.

Conclusions: Treatment regimens among people with ID tended to include older types of medication compared with what was prescribed in the general population. To ensure that this is medically appropriate and not due to failure to update the treatment regimen, it is important to investigate if the people with ID and diabetes mellitus or hypertension are subjected to the same regular drug reviews that are recommended for older adults in general.

Keywords: Aged, Cardiovascular disease risk factors, Drug prescription, Middle-aged

\footnotetext{
* Correspondence: anna.axmon@med.lu.se

'Division of Occupational and Environmental Medicine, Faculty of Medicine,

Lund University, SE-221 00 Lund, Sweden

${ }^{2}$ Department of Health Sciences, Faculty of Medicine, Lund University, Lund,

Sweden

Full list of author information is available at the end of the article
} 


\section{Background}

Cardiovascular disease (CVD), such as myocardial infarction and stroke, is the leading noncommunicable disease as well as the most common cause of death in the world [1]. Alongside several lifestyle factors, hypertension and diabetes mellitus have been found to be risk factors for CVD. Thus, reductions of these disorders should reasonably lead to reductions in CVD and related deaths.

In the general population [2-4] as well as among people with intellectual disability (ID) [5-9], increasing age is a risk factor for both hypertension and diabetes mellitus. Adults with ID seem to experience higher rates of diabetes mellitus [10-13] than people without ID. If this effect is found also in older age groups, i.e. if the age effect is similar in people with and without ID, has been less studied. The few results published so far suggest that the increase in diabetes mellitus risk among people with ID compared to the general population decrease with age [12], which translates into a smaller age effect among people with ID. With respect to hypertension and ID, existing literature points towards similar rates among people with ID and in the general population [7, 8, 14], although this may be an effect of decreased rates among men and increased rates among women [7]. Nevertheless, studies focusing on older adults with ID are scarce and as they may constitute a group particularly burdened by diseases that are risk factors for CVD, further knowledge is needed in this area.

There is a variety of pharmacological treatments for diabetes mellitus, each associated with its own potential side effects [15]. Decision on treatment regime is facilitated by a range of guidelines [16-19]. However, in older patient groups, the guidelines for younger populations may not be relevant. For example, the use of metformin - which is the primary choice of drug in younger people - is often limited for older people because of comorbid conditions such as chronic renal insufficiency and heart failure [20]. To the best of our knowledge, treatment use among older people with ID and diabetes mellitus has not yet been described in scientific literature.

Unlike for diabetes mellitus, hypertension can be treated with the same drugs in older people as in younger age groups [21, 22]. Based on a high prevalence, van de Louw et al. [8] called for people with ID and hypertension to be treated in the same manner as in the general population, following national guidelines. However, as far as we know, there are no data published regarding actual treatments used among older people with ID and hypertension. Indeed, only a few years ago, de Winter et al. [7] concluded that a policy on prevention, detection and treatment of risk factors for CVD among people with ID was urgently needed.

In order to get health policies in place for older people with ID - a vulnerable group in which many have a lifelong experience of difficulties in expressing their illnesses and health care needs - increased knowledge is essential. Not only knowledge concerning the prevalence of disorders and their treatment per se, but also in comparison with age peers in the general population. Such information could play a vital role in determining which types of prophylactic health practices should be used to delay the onset and minimize the occurrence of life threatening CVD. Therefore, the aim of this study was to describe and compare prevalence ratios of diabetes mellitus and hypertension between older adults with ID and their age peers in the general population, and to describe and compare treatment patterns in these two groups. Moreover, to investigate differences in prevalence ratios stratified by sex, i.e. separately for men and women, as well as assess possible sex differences within each cohort.

\section{Methods}

This is a register based study, using Swedish national registers both to define the original cohorts, identify the subcohorts with diagnosis of diabetes mellitus or hypertension, and collect information on outcomes (drug prescriptions).

\section{Registers}

The LSS register contains information on all support and service provided according to the Act Concerning Support and Service for Persons with Certain Functional Impairments [23] (Swedish abbreviation: LSS). According to this act, a person with ID or autism spectrum disorder (ASD) may apply to his/her municipality for the support needed to optimize his/her opportunity to achieve good living conditions in his/her daily life. The act lists ten measures of support, of which eight are provided for adults. These include e.g. daytime activities, personal assistant, and special housing. The latter is in the form of private apartments with access to common areas. For those with less need of assistance, the common areas are normally in the proximity to the apartments, and staff is accessible around the clock, whereas for those with higher need of assistance, the common areas are adjoining the apartments and staff is on site at all times.

Although a diagnosis of either ID or ASD is required to receive the support, the diagnosis itself is not recorded in the register.

The National Patient Register contains records of all inpatient and outpatient specialist visits in Sweden. Inpatient visits are recorded at the date of discharge. For each visit, one primary and up to 21 secondary diagnoses are recorded, coded according to the International Statistical Classification of Diseases and Related Health Problems 10th Revision (ICD-10). 
The Prescribed Drug Register contains information on all purchases of prescribed drugs in Sweden, corresponding to $84 \%$ of the total volume of drugs, starting July 2005 [24]. Purchases are registered at the date of dispensation, and information on the drug is given according to the Anatomical Therapeutic Chemical (ATC) classification system.

\section{Study cohorts}

Information about the two original cohorts has been given in detail elsewhere [25-27]. Briefly, an administrative cohort of people with ID $(n=7936)$ was established using the LSS register. Moreover, a referent cohort was randomly selected from the general Swedish population by Statistics Sweden, one-to-one matched on sex and year of birth (gPop). All people in both cohorts were 55 + years old and alive at the end of 2012. For the ID cohort, information on residence in special housing, as described above, was collected from the LSS register, and was categorized as having lived in special housing at least one year or never during the study period.

\section{Diagnoses}

Data regarding all diagnoses (primary as well as secondary) in inpatient care and at outpatient specialist visits during 2006-2012 were collected from the Swedish National Patient Register. Diagnostic codes E10 (insulin-dependent diabetes mellitus, or type 1 diabetes), E11 (non-insulindependent diabetes mellitus, or type 2 diabetes), E12 (malnutrition-related diabetes mellitus), E13 (other specified diabetes mellitus), and E14 (unspecified diabetes mellitus) were considered as diabetes mellitus, and I10 (essential [primary] hypertension), I11 (hypertensive heart disease), I12 (hypertensive renal disease), I13 (hypertensive heart and renal disease), and I15 (secondary hypertension) were considered as hypertension. All diagnoses made during the study period are listed in Additional file 1.

\section{Drug prescriptions}

Information on purchases of prescribed drugs was collected for the period 2006-2012 from the Swedish Prescribed Drug Register. Drugs investigated as drugs for diabetes mellitus were those with ATC code A10. These were aggregated into third (e.g. A10B, blood glucose lowering drugs excluding insulins) and fourth (e.g. A10BA, biguanides) ATC level groups. As drugs for hypertension we considered those with ATC codes $\mathrm{C} 03$ (diuretics), C07 (beta blocking agents), C08 (calcium channel blockers), and C09 (agents acting on the reninangiotensin system). These were analyzed separately, and also aggregated to ACE (Angiotensin-Converting-Enzyme) inhibitors (C09A \& C09B) and angiotensin II antagonists (C09C \& C09D). All drugs prescribed during the study period are listed in Additional file 2.

\section{Statistics}

Analyses of dichotomous outcomes (e.g. having at least one prescription) were performed comparing people with ID to the gPop sample using generalized linear models (GLM) with a Poisson distribution, log link function and robust covariance matrix estimator, thus estimating relative risks (RRs) with 95\% confidence intervals (CIs). Secondary analyses were performed comparing people with ID to the gPop sample stratified by sex. Moreover, we investigated sex differences in drug prescription within each cohort. Statistical interaction was assessed by introducing the cross product of sex and cohort affiliation in separate models.

All analyses regarding diabetes mellitus were stratified by type of diabetes mellitus, including only those with at least one diagnosis of either insulin- or noninsulin-dependent diabetes mellitus. Thus, 44 people with diagnosis of unspecified diabetes mellitus only, and one person with diagnosis of other specified diabetes mellitus were excluded.

All analyses were performed in SPSS version 23.0. $P$ values below 0.05 were considered statistically significant. Analyses were only performed when both groups to be compared contained at least five people.

\section{Results}

\section{Disease prevalence}

As both diabetes mellitus and hypertension are chronic disorders, the prevalence at the end of the study can be estimated by using the number of diagnoses and prescriptions during the study period.

For all proxies of diabetes mellitus (combinations of prescription and diagnosis), the people in the ID cohort had a higher prevalence compared with the gPop cohort (Table 1). This was driven by a higher prevalence among women, as no difference in occurrence of diabetes mellitus was found among the men. Within the gPop cohort, a higher risk of diabetes mellitus diagnosis was found for men (RR 1.64 [1.39-1.95]). The same effect was not found in the ID cohort (0.93 [0.81-1.08]).

A higher prevalence for the ID cohort was also found for non-insulin-dependent diabetes mellitus, but not for insulin-dependent diabetes mellitus (Table 1). Within the gPop cohort, men had a higher risk than women of non-insulin-dependent (RR 1.61 [1.35-1.93]) as well as insulin-dependent diabetes mellitus (1.33 [0.98-1.81]), although statistical significance was not achieved for the latter. No statistically significant differences were found between sexes in the ID cohort, neither for non-insulin-dependent (men vs women 0.95 [0.81-1.10]) nor insulin-dependent diabetes mellitus (1.11 [0.83-1.29]). Special housing was associated with a lower risk of both non-insulin-dependent (0.70 [0.59-0.83]) 


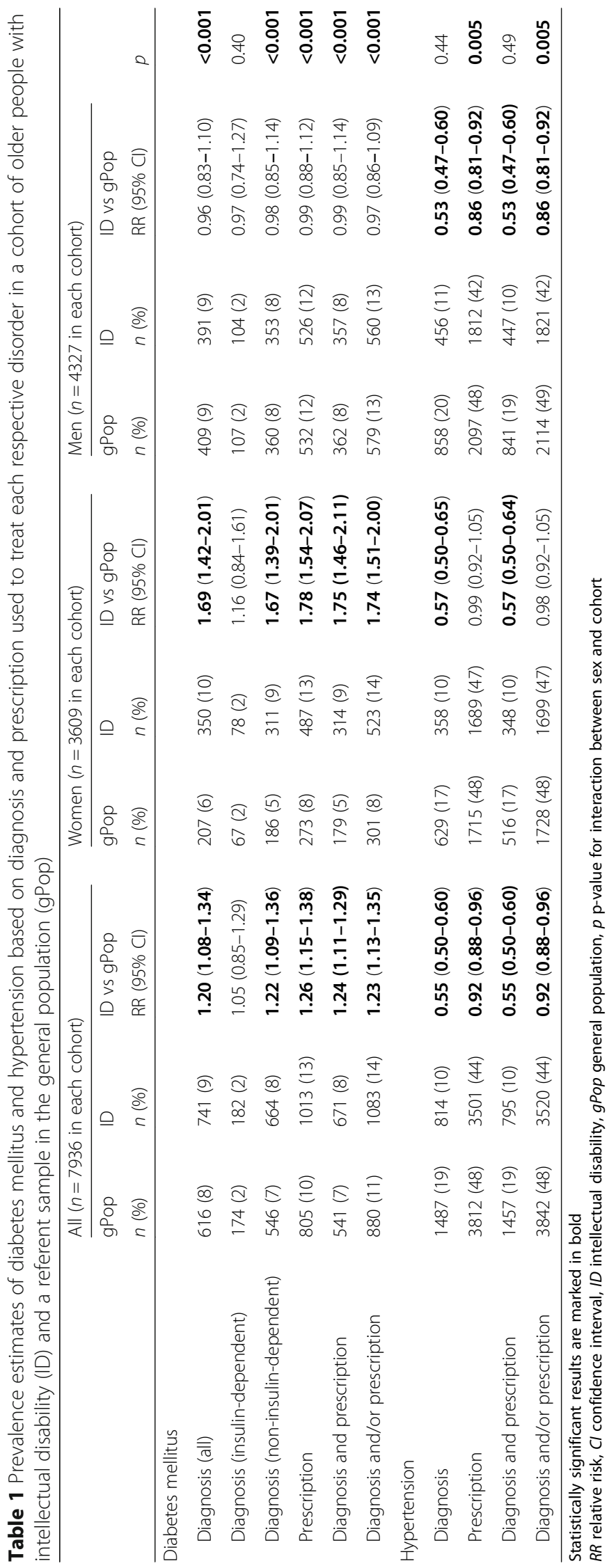


Table 2 Prescription of drugs used for diabetes mellitus and hypertension among those with at least one such diagnosis in a group of people with intellectual disability (ID) and referents from the general population (gPop)

\begin{tabular}{|c|c|c|c|}
\hline & \multicolumn{3}{|l|}{ All } \\
\hline & gPop & ID & ID vs gPop \\
\hline & $n(\%)$ & n (\%) & $\mathrm{RR}(95 \% \mathrm{Cl})$ \\
\hline Insulin-dependent diabetes mellitus & $n=174$ & $n=182$ & \\
\hline Insulins and analogues & $147(84)$ & $155(85)$ & $1.01(0.80-1.26)$ \\
\hline Fast-acting & $103(59)$ & $80(44)$ & $0.74(0.55-0.99)$ \\
\hline Intermediate-acting & $71(41)$ & $57(31)$ & $0.77(0.56-1.09)$ \\
\hline Intermediate/long-acting comb. fast-acting & $52(30)$ & $96(53)$ & $1.77(1.26-2.47)$ \\
\hline Long-acting & $65(37)$ & $62(34)$ & $0.91(0.64-1.29)$ \\
\hline Blood glucose lowering drugs excl. Insulins & $96(55)$ & $126(69)$ & $1.26(0.96-1.64)$ \\
\hline Biguanides & $91(52)$ & $112(62)$ & $1.18(0.89-1.55)$ \\
\hline Sulfonylureas & $27(16)$ & $55(30)$ & $1.95(1.23-3.09)$ \\
\hline Combinations & $4(2)$ & $2(1)$ & NC \\
\hline a glucosidase inhibitors & $3(2)$ & $4(2)$ & NC \\
\hline Thiazolidinediones & $5(3)$ & $3(2)$ & NC \\
\hline Dipeptidyl peptidase-4 inhibitors & $8(5)$ & $2(1)$ & NC \\
\hline Repaglinide or nateglinide & $10(6)$ & $7(4)$ & $0.67(0.26-1.76)$ \\
\hline Exenatide or liraglutide & $1(1)$ & $3(2)$ & NC \\
\hline Non-insulin-dependent diabetes & $n=546$ & $n=664$ & \\
\hline Insulins and analogues & $252(46)$ & $338(51)$ & $1.10(0.94-1.30)$ \\
\hline Fast-acting & $125(23)$ & $136(20)$ & $0.90(0.70-1.14)$ \\
\hline Intermediate-acting & $131(24)$ & $129(19)$ & $0.81(0.64-1.03)$ \\
\hline Intermediate/long-acting comb. fast-acting & $114(21)$ & $212(32)$ & $1.53(1.22-1.92)$ \\
\hline Long-acting & $84(15)$ & $100(15)$ & $0.98(0.73-1.31)$ \\
\hline Blood glucose lowering drugs excl. Insulins & $423(77)$ & $528(80)$ & $1.03(0.90-1.17)$ \\
\hline Biguanides & $398(73)$ & $473(71)$ & $0.98(0.86-1.12)$ \\
\hline Sulfonylureas & $150(27)$ & $230(35)$ & $1.26(1.03-1.55)$ \\
\hline Combinations & $20(4)$ & $11(2)$ & $0.45(0.22-0.94)$ \\
\hline a glucosidase inhibitors & $6(1)$ & $8(1)$ & $1.10(0.38-3.16)$ \\
\hline Thiazolidinediones & $23(4)$ & $21(3)$ & $0.75(0.42-1.36)$ \\
\hline Dipeptidyl peptidase-4 inhibitors & $42(8)$ & $2(3)$ & $0.43(0.26-0.72)$ \\
\hline Repaglinide or nateglinide & $34(6)$ & $36(5)$ & $0.87(0.55-1.39)$ \\
\hline Exenatide or liraglutide & $15(3)$ & $7(1)$ & $0.38(0.16-0.94)$ \\
\hline Hypertension & $n=1487$ & $n=814$ & \\
\hline Diuretics & $641(43)$ & $482(59)$ & $1.37(1.22-1.55)$ \\
\hline Beta blocking agents & $953(64)$ & $497(61)$ & $0.95(0.86-1.06)$ \\
\hline Calcium channel blockers & $792(53)$ & $362(44)$ & $0.84(0.74-0.95)$ \\
\hline Agents acting on the renin-angiotensin system & $1184(80)$ & $543(67)$ & $0.84(0.75-0.93)$ \\
\hline ACE inhibitors & $863(58)$ & $463(57)$ & $0.98(0.88-1.10)$ \\
\hline Angiotensin II antagonists & $614(41)$ & $145(18)$ & $0.43(0.36-0.52)$ \\
\hline
\end{tabular}

Statistically significant results are marked in bold

$R R$ relative risk, $\mathrm{Cl}$ confidence interval, $I D$ intellectual disability, gPop general population, $N C$ not calculated due to too few observations

and insulin-dependent diabetes mellitus (0.80 [0.571.11]), although the latter was not statistically significant.
People with ID were less likely to have hypertension than the people in the gPop sample (Table 1). This was true for all proxies (combinations of prescription and 
diagnosis) of hypertension when examining the whole cohorts. However, when stratifying by sex, proxies including prescription of hypertension drugs was not associated with a higher prevalence for women with ID compared to women in the gPop cohort. Within the gPop cohort, men were statistically significantly more likely than women to have a diagnosis of hypertension (RR 1.14 [1.03-1.26]). The corresponding sex difference was not found in the ID cohort (men vs women 1.06 [0.93-1.22]). However, within the ID cohort, special housing was associated with a lower risk of being diagnosed with hypertension (0.70 [0.60-0.82]).

\section{Prescription of drugs used in diabetes mellitus}

In the ID cohort, $91 \%$ of those with diagnosis of diabetes mellitus had at least one prescription of drugs for diabetes mellitus, and $66 \%$ of those with at least one prescription had a diagnosis (Table 1). The corresponding numbers in the gPop cohort were $88 \%$ and $67 \%$.

For both types of diabetes mellitus, people with ID were more likely than those in the gPop sample to be prescribed intermediate or long-acting insulins combined with fast-acting insulins as well as sulfonylureas (Table 2, Additional file 3). They were, however, less likely to be prescribed fast-acting insulins and analogues for insulin-dependent diabetes mellitus. Also, they were less likely to be prescribed combinations of oral blood glucose lowering drugs, dipeptidyl peptidase-4, and exenatide/liraglutide for non-insulin-dependent diabetes mellitus. The pattern of prescription (ID vs gPop) of the investigated drugs were similar for men and women. Furthermore, there were no differences between women and men in neither cohort (Table 3).

Among those with insulin-dependent diabetes mellitus within the ID cohort, people in special housing were more likely to be prescribed fast- (RR 1.25 [0.73-2.14]), intermediate- (1.80 [0.89-3.68]), and long-acting (1.06 [0.59-1.90]) insulins and analogues for injection, as well as sulfonylureas (1.52 [0.77-3.02]). They were less likely to be prescribed insulins and analogues (0.94 [95\% CI 0.66-1.34]), intermediate- or long-acting combined with fast-acting insulins and analogues for injection (0.78 [0.51-1.21]), blood glucose lowering drugs excluding insulins (0.95 [0.64-1.42], and biguanides (0.88 [0.581.34]). None of these results were, however, statistically significant. Other drugs were not investigated due to too low numbers.

Among those with non-insulin-dependent diabetes mellitus within the ID cohort, people in special housing were more likely to be prescribed intermediate-acting insulins and analogues for injection (RR 1.21 [0.81-1.82]) and sulfonylureas (1.11 [0.83-1.49]). They were less likely to have been prescribed insulins and analogues (0.98 [95\% CI 0.68-1.07]), fast- (0.99 [0.68-1.44]) and long-acting (0.78 [0.51-1.18]) insulins and analogues for injection, intermediate- or long-acting combined with fast-acting insulins and analogues for injection (0.83 [0.62-1.11]), blood glucose lowering drugs excluding insulins (0.97 [0.80-1.17], and biguanides (0.92 [0.761.12]). None of these results were, however, statistically significant. Other drugs were not investigated due to too low numbers.

\section{Prescription of drugs for hypertension}

In the ID cohort, $98 \%$ of those with diagnosis of hypertension had at least one prescription of drugs to treat hypertension, and $23 \%$ of those with at least one prescription had a diagnosis (Table 1). The corresponding numbers in the gPop cohort were $98 \%$ and $38 \%$.

Among those with at least one diagnosis of hypertension, a higher percentage among people with ID than in the gPop sample were prescribed diuretics at least once during the study period (Table 2). The opposite, i.e. lower percentage of people with prescription among people with ID, was seen for calcium channel blockers and agents acting on the renin-angiotensin system. The latter was driven by a lower prescription of angiotensin II antagonists in the ID cohort. The patterns were similar when stratifying by sex. Within the gPop cohort, men were less likely than women to be prescribed diuretics, and more likely to be prescribed agents acting on the renin-angiotensin system, specifically ACE inhibitors (Table 3). No sex differences were found within the ID cohort. However, special housing was associated with statistically significantly lower risks of prescription of beta blocking agents (RR 0.75 [95\% CI $0.67-0.84]$ ), calcium channel blockers $(0.70$ [0.60-0.81]), agents acting on the renin-angiotensin system (0.67 [0.61-0.76]), ACE inhibitors (0.68 [0.60-0.77]), and angiotensin II antagonists $(0.61[0.49-0.75])$, but not diuretics (1.04 [0.94$1.16])$.

\section{Discussion}

Women, but not men, with ID were more likely than the general population to have diabetes mellitus, regardless of proxy (diagnosis or prescription of drugs) for disorder. When determining presence of hypertension based on diagnosis, both men and women with ID were less likely to have hypertension than the general population. However, when considering prescription of drugs as a proxy for hypertension, the reduced prevalence was found only among men.

The major strength of the present study is the use of a national register for outcome assessments. In Sweden, all drugs prescribed for both diabetes mellitus and hypertension are sold by prescription only. Thus, no misclassification has been introduced by over-thecounter purchases. 
Table 3 Prescription of drugs used for diabetes mellitus and hypertension among those with at least one such diagnosis in a group of people with intellectual disability (ID) and referents from the general population (gPop), stratified by sex

\begin{tabular}{|c|c|c|c|c|}
\hline & & gPop & ID & ID vs gPop \\
\hline & & $n(\%)$ & $n(\%)$ & $\operatorname{RR}(95 \% \mathrm{Cl})$ \\
\hline Insulin-dependent diabetes mellitus & & & & \\
\hline Insulins and analogues & Women & $57(85)$ & $65(83)$ & $0.98(0.69-1.40)$ \\
\hline & Men & $90(84)$ & $90(87)$ & $1.03(0.77-1.38)$ \\
\hline & Men vs women & $0.99(0.71-1.38)$ & $1.04(0.76-1.43)$ & \\
\hline Fast-acting & Women & $42(63)$ & $30(38)$ & $0.61(0.38-0.98)$ \\
\hline & Men & $61(57)$ & $50(48)$ & $0.84(0.58-1.23)$ \\
\hline & Men vs women & $0.91(0.61-1.35)$ & $1.25(0.80-1.97)$ & \\
\hline Intermediate-acting & Women & $24(36)$ & $21(27)$ & $0.75(0.42-1.35)$ \\
\hline & Men & $47(44)$ & $36(35)$ & $0.79(0.51-1.22)$ \\
\hline & Men vs women & $1.23(0.75-2.01)$ & $1.29(0.75-2.20)$ & \\
\hline Intermediate/long-acting comb. fast-acting & Women & $22(33)$ & $42(54)$ & $1.64(0.98-2.75)$ \\
\hline & Men & $30(28)$ & $54(52)$ & $1.85(1.19-2.89)$ \\
\hline & Men vs women & $0.85(0.49-1.48)$ & $0.96(0.64-1.44)$ & \\
\hline Long-acting & Women & $29(43)$ & $25(32)$ & $0.74(0.43-1.26)$ \\
\hline & Men & $36(34)$ & $37(36)$ & $1.06(0.69-1.67)$ \\
\hline & Men vs women & $0.78(0.48-1.27)$ & $1.11(0.67-1.84)$ & \\
\hline Blood glucose lowering drugs excl. Insulins & Women & $40(60)$ & $52(67)$ & $1.12(0.74-1.69)$ \\
\hline & Men & $56(52)$ & $74(71)$ & $1.36(0.96-1.92)$ \\
\hline & Men vs women & $0.88(0.58-1.32)$ & $1.07(0.75-1.52)$ & \\
\hline Biguanides & Women & $37(55)$ & $46(59)$ & $1.07(0.69-1.65)$ \\
\hline & Men & $54(50)$ & $66(63)$ & $1.26(0.88-1.80)$ \\
\hline & Men vs women & $0.91(0.60-1.39)$ & $1.08(0.74-1.57)$ & \\
\hline Sulfonylureas & Women & $13(19)$ & $21(27)$ & $1.39(0.70-2.77)$ \\
\hline & Men & $14(13)$ & $34(33)$ & $2.50(1.34-4.66)$ \\
\hline & Men vs women & $0.67(0.32-1.44)$ & $1.21(0.71-2.09)$ & \\
\hline Combinations & Women & $1(1)$ & $2(3)$ & NC \\
\hline & Men & $3(3)$ & $0(0)$ & NC \\
\hline & Men vs women & NC & NC & \\
\hline a glucosidase inhibitors & Women & $2(3)$ & $3(4)$ & NC \\
\hline & Men & $1(1)$ & $1(1)$ & NC \\
\hline & Men vs women & NC & NC & \\
\hline Thiazolidinediones & Women & $3(4)$ & $0(0)$ & NC \\
\hline & Men & $2(2)$ & $3(3)$ & NC \\
\hline & Men vs women & NC & NC & \\
\hline Dipeptidyl peptidase-4 inhibitors & Women & $3(4)$ & $2(3)$ & NC \\
\hline & Men & $5(5)$ & $0(0)$ & NC \\
\hline & Men vs women & NC & NC & \\
\hline Repaglinide or nateglinide & Women & $3(4)$ & $4(5)$ & NC \\
\hline & Men & $7(7)$ & $3(3)$ & NC \\
\hline & Men vs women & NC & NC & \\
\hline
\end{tabular}


Table 3 Prescription of drugs used for diabetes mellitus and hypertension among those with at least one such diagnosis in a group of people with intellectual disability (ID) and referents from the general population (gPop), stratified by sex (Continued)

\begin{tabular}{|c|c|c|c|c|}
\hline \multirow{3}{*}{ Exenatide or liraglutide } & & \multirow{2}{*}{$\begin{array}{l}\text { gPop } \\
n(\%)\end{array}$} & \multirow{2}{*}{$\begin{array}{l}\text { ID } \\
n(\%)\end{array}$} & \multirow{2}{*}{$\begin{array}{l}\text { ID vs gPop } \\
\text { RR ( } 95 \% \text { Cl) }\end{array}$} \\
\hline & & & & \\
\hline & Women & $0(0)$ & $1(1)$ & NC \\
\hline & Men & $1(1)$ & $2(2)$ & NC \\
\hline & Men vs women & NC & NC & \\
\hline \multicolumn{5}{|l|}{ Non-insulin-dependent diabetes } \\
\hline \multirow[t]{3}{*}{ Insulins and analogues } & Women & $94(51)$ & $161(52)$ & $1.02(0.79-1.32)$ \\
\hline & Men & $158(44)$ & $177(50)$ & $1.14(0.92-1.42)$ \\
\hline & Men vs women & $0.87(0.67-1.12)$ & $0.97(0.78-1.20)$ & \\
\hline \multirow[t]{3}{*}{ Fast-acting } & Women & $53(28)$ & $61(20)$ & $0.69(0.48-1.00)$ \\
\hline & Men & $72(20)$ & $75(21)$ & $1.06(0.77-1.47)$ \\
\hline & Men vs women & $0.70(0.49-1.00)$ & $1.08(0.77-1.52)$ & \\
\hline \multirow[t]{3}{*}{ Intermediate-acting } & Women & $48(26)$ & $68(22)$ & $0.85(0.59-1.23)$ \\
\hline & Men & $83(23)$ & $61(17)$ & $0.75(0.54-1.04)$ \\
\hline & Men vs women & $0.89(0.63-1.28)$ & $0.79(0.56-1.12)$ & \\
\hline \multirow[t]{3}{*}{ Intermediate/long-acting comb. fast-acting } & Women & $44(24)$ & $103(33)$ & $1.40(0.98-1.99)$ \\
\hline & Men & $70(19)$ & $109(31)$ & $1.59(1.18-2.14)$ \\
\hline & Men vs women & $0.82(0.56-1.20)$ & $0.93(0.71-1.22)$ & \\
\hline \multirow[t]{3}{*}{ Long-acting } & Women & $36(19)$ & $40(13)$ & $0.67(0.42-1.04)$ \\
\hline & Men & $48(13)$ & $60(17)$ & $1.28(0.87-1.86)$ \\
\hline & Men vs women & $0.69(0.45-1.06)$ & $1.32(0.89-1.97)$ & \\
\hline \multirow[t]{3}{*}{ Blood glucose lowering drugs excl. Insulins } & Women & $138(74)$ & $246(79)$ & $1.07(0.87-1.31)$ \\
\hline & Men & $285(79)$ & $282(80)$ & $1.01(0.86-1.19)$ \\
\hline & Men vs women & $1.07(0.87-1.31)$ & $1.01(0.85-1.20)$ & \\
\hline \multirow[t]{3}{*}{ Biguanides } & Women & $127(68)$ & $225(72)$ & $1.06(0.85-1.32)$ \\
\hline & Men & $271(75)$ & $248(70)$ & $0.93(0.79-1.11)$ \\
\hline & Men vs women & $1.10(0.89-1.26)$ & $0.97(0.81-1.16)$ & \\
\hline \multirow[t]{3}{*}{ Sulfonylureas } & Women & $51(27)$ & $105(34)$ & $1.23(0.88-1.72)$ \\
\hline & Men & $99(28)$ & $125(35)$ & $1.29(0.99-1.68)$ \\
\hline & Men vs women & $1.00(0.72-1.41)$ & $1.05(0.81-1.36)$ & \\
\hline \multirow[t]{3}{*}{ Combinations } & Women & $7(4)$ & $7(2)$ & $0.60(0.21-1.71)$ \\
\hline & Men & $13(4)$ & $4(1)$ & NC \\
\hline & Men vs women & $0.96(0.38-2.41)$ & NC & \\
\hline \multirow[t]{3}{*}{ a glucosidase inhibitors } & Women & $4(2)$ & $7(2)$ & NC \\
\hline & Men & $2(1)$ & $1(0)$ & NC \\
\hline & Men vs women & NC & NC & \\
\hline \multirow[t]{3}{*}{ Thiazolidinediones } & Women & $7(4)$ & $7(2)$ & $0.60(0.21-1.71)$ \\
\hline & Men & $16(4)$ & $14(4)$ & $0.89(0.44-1.83)$ \\
\hline & Men vs women & $1.18(0.49-2.87)$ & $1.76(0.71-4.37)$ & \\
\hline \multirow[t]{3}{*}{ Dipeptidyl peptidase-4 inhibitors } & Women & $17(9)$ & $13(4)$ & $0.46(0.22-0.94)$ \\
\hline & Men & $25(7)$ & $9(3)$ & $0.36(0.17-0.79)$ \\
\hline & Men vs women & $0.76(0.41-1.41)$ & $0.61(0.26-1.43)$ & \\
\hline
\end{tabular}


Table 3 Prescription of drugs used for diabetes mellitus and hypertension among those with at least one such diagnosis in a group of people with intellectual disability (ID) and referents from the general population (gPop), stratified by sex (Continued)

\begin{tabular}{|c|c|c|c|c|}
\hline & & gPop & ID & ID vs gPop \\
\hline & & $n(\%)$ & $n(\%)$ & RR $(95 \% \mathrm{Cl})$ \\
\hline \multirow[t]{3}{*}{ Repaglinide or nateglinide } & Women & $11(6)$ & $20(6)$ & $1.09(0.52-2.27)$ \\
\hline & Men & $23(6)$ & $16(5)$ & $0.71(0.38-1.34)$ \\
\hline & Men vs women & $1.08(0.53-2.22)$ & $0.71(0.37-1.36)$ & \\
\hline \multirow[t]{3}{*}{ Exenatide or liraglutide } & Women & $3(2)$ & $5(2)$ & NC \\
\hline & Men & $12(3)$ & $2(1)$ & NC \\
\hline & Men vs women & NC & NC & \\
\hline \multicolumn{5}{|l|}{ Hypertension } \\
\hline \multirow[t]{3}{*}{ Diuretics } & Women & 307 (49) & $220(61)$ & $1.26(1.06-1.50)$ \\
\hline & Men & 334 (39) & $262(57)$ & $1.48(1.26-1.74)$ \\
\hline & Men vs women & $0.80(0.68-0.93)$ & $0.94(0.78-1.12)$ & \\
\hline \multirow[t]{3}{*}{ Beta blocking agents } & Women & $415(66)$ & $216(60)$ & $0.91(0.78-1.08)$ \\
\hline & Men & $538(63)$ & $281(62)$ & $0.98(0.85-1.14)$ \\
\hline & Men vs women & $0.95(0.84-1.08)$ & $1.02(0.86-1.22)$ & \\
\hline \multirow[t]{3}{*}{ Calcium channel blockers } & Women & $317(50)$ & $148(41)$ & $0.82(0.68-1.00)$ \\
\hline & Men & $475(55)$ & $214(47)$ & $0.85(0.72-1.00)$ \\
\hline & Men vs women & $1.10(0.95-1.27)$ & $1.14(0.92-1.40)$ & \\
\hline \multirow{3}{*}{$\begin{array}{l}\text { Agents acting on the renin-angiotensin } \\
\text { system }\end{array}$} & Women & $455(72)$ & $223(62)$ & $0.86(0.73-1.01)$ \\
\hline & Men & 729 (85) & $320(70)$ & $0.83(0.72-0.94)$ \\
\hline & Men vs women & $1.18(1.05-1.32)$ & $1.13(0.95-1.34)$ & \\
\hline \multirow[t]{3}{*}{ ACE inhibitors } & Women & $319(51)$ & $189(53)$ & $1.04(0.87-1.25)$ \\
\hline & Men & $544(63)$ & $274(60)$ & $0.95(0.82-1.10)$ \\
\hline & Men vs women & $1.25(1.09-1.44)$ & $1.14(0.95-1.37)$ & \\
\hline \multirow[t]{3}{*}{ Angiotensin II antagonists } & Women & $259(41)$ & $60(17)$ & $0.41(0.31-0.54)$ \\
\hline & Men & $355(41)$ & $85(19)$ & $0.45(0.36-0.57)$ \\
\hline & Men vs women & $1.01(0.86-1.18)$ & $1.11(0.80-1.55)$ & \\
\hline
\end{tabular}

Statistically significant results are marked in bold.

$R R$ relative risk, $\mathrm{Cl}$ confidence interval, ID intellectual disability, $g P o p$ general population, NC not calculated due to too few observations

We used the National Patient Register to identify people with a diagnosis of diabetes mellitus or hypertension. This register contains only diagnoses made in inpatient care and at outpatient specialist visits, not diagnoses made in primary care. In Sweden, the majority of those with insulin-dependent diabetes mellitus is treated at specialist centers, whereas most of the people with non-insulin-dependent diabetes mellitus are treated in primary care, with the exception of the more severe and complex cases [28]. In the present study, this is reflected in that only two thirds of those with prescription of drugs for diabetes mellitus had a recorded diagnosis of diabetes mellitus. That the percentage is similar in both the ID and the gPop cohort suggests that the failure to include those with diagnosis only in primary care have not biased the results when comparing the two cohorts. However, a similar percentage would also occur if people with ID have a higher prevalence of diabetes mellitus but are more likely to be undiagnosed and/or untreated, e.g. due to differences in access to primary care. This must be taken into consideration when interpreting the patterns of drug prescriptions in the different cohorts. Moreover, it must be acknowledged that the people included in the two cohorts may not be representative of all people with diabetes mellitus, and that the results might be generalizable only to people with severe or complex types of diabetes mellitus.

A potential weakness with the present study is the inability to adjust for potential confounders, as information on such were not available in any of the registers used. Although sex and age were taken into account in the matching of the referent cohort, we were not able to consider other factors that are known to be risk or protective factors for diabetes mellitus or hypertension, as well as differing in prevalence among people with ID and the general population. These include e.g. body 
mass index (BMI) and obesity [29-31], smoking [30, 32, 33], and alcohol use [30, 34, 35].

People in the ID cohort had a higher prevalence of diabetes mellitus (overall, i.e. not stratified by insulindependence), regardless of which proxy was used. This was driven by a high prevalence among the women with ID. Sex comparisons within each cohort and cohort comparisons within each sex revealed that women from the general population had a lower prevalence of diabetes mellitus than the other three groups. A different way to put this is that women with ID did not have the prevalence expected for women, but rather the same prevalence as found among the men. Women with ID are at greater risk of obesity and overweight than men with ID [36]. As a high BMI is one of the major risk factors for diabetes mellitus, a high prevalence of overweight among women with ID may be an explanation for their higher prevalence of non-insulin-dependent diabetes mellitus.

The prescription patterns were similar among those with insulin-dependent and non-insulin-dependent diabetes mellitus, in that people with ID were more likely to be prescribed combination drugs and sulfonylureas. Combination drugs are easier to handle than single drugs, both for the person with ID and for his or her carer. However, they also decrease the flexibility in adjusting the treatment based on individual needs. People with ID were also more likely to be prescribed sulfonylureas. For non-insulin dependent diabetes mellitus, the Swedish Medical Products Agency, recommend metformin, a biguanide, as first-line treatment [37]. Sulfonylurea, which is an older drug, should only be used when there is a contra-indication or intolerance for metformin. Our assessment of prescriptions is based on data for the entire study period, i.e. 20062012. Thus, our data would be in agreement with a pattern of changing from sulfonylurea to metformin during the study period. However, when looking at yearly prescriptions rather than prescriptions for the whole study period, there is no obvious trend over time (data not shown). Thus, the results for treatment of diabetes mellitus suggest that people with ID to a greater extent than the general population are prescribed drugs which are not recommended by the Medical Products Agency. At the same time, use of metformin is often limited for older people, due to comorbid conditions [20]. People with ID tend to have an earlier onset of the aging process [5,38], and use of metformin may therefore be inappropriate at younger ages than in the general population.

When using only proxies for hypertension depending on diagnosis, people with ID had an almost halved risk of having hypertension as people in the general population, a result that was consistent for both men and women. This is opposite to what we expected, partly as previous studies have reported similar rates of hypertension among people with ID and the general population [8], and partly since people with ID display several risk factors associated with hypertension, such as congenital heart disease $[39,40]$ and obesity $[36,41]$. When including also prescription of drugs to treat hypertension in the proxy for the disorder, the results were somewhat closer to what we had anticipated. The discrepancy between hypertension defined by prescription and hypertension defined by diagnosis is not surprising, as drugs used to treat hypertension also have other indications. For example, diuretics may be used to treat edema, and beta-blocking agents may be used to treat migraine. However, it is noteworthy that among people with ID, the fraction with diagnosis among those with prescription was lower than in the gPop cohort. This could either be due to a higher prescription rate among those without hypertension among people with ID, or to differences in which type of care (primary, inpatient, outpatient specialist) diagnoses of hypertension are made. If the cause is the latter, i.e. if people with ID are more likely than the general population to get their hypertension diagnosis in primary care than in other types of care, this could explain the lower prevalence of hypertension found among people with ID in the present study. However, another reason may be that people with ID are underdiagnosed with respect to hypertension. This could happen e.g. if either they themselves or their carers fail to understand the signs of hypertension or communicate them to the physician, and if the physician due to this fails to make a correct diagnosis. Underdiagnosis would also occur if routine blood pressure checks are not made on older people with ID to the same extent as in the general population.

Among those with at least one diagnosis of hypertension, people with ID were more likely to be prescribed diuretics and less likely to be prescribed several of the other drugs investigated. Another way of putting it, is that people with ID are more likely to be prescribed older drugs, whereas newer alternatives are more often used in the general population. There may be several reasons for this. People without ID probably have more knowledge about their disease and treatment options than people with ID. Thereby, they can make higher demands on their physician to try newer treatment regimes. They may also be more aware of possible adverse effects and alert their physician to them and thereby get a different drug. With respect to people with ID, it is not unlikely that a physician who have prescribed a drug that improves the health of the patient is unwilling to change the treatment simply because newer options are available. Although newer drugs are not by default better than older ones, 
especially in specific patient groups such as older people with ID, difficulties for people with ID in recognizing and communicating adverse effects warrant monitoring and regular drug reviews in this group.

\section{Conclusions}

As expected, people with ID had a higher prevalence of diabetes mellitus than the general population. There were also differences in treatment regimes, in that people with ID more often were prescribed less flexible but more easy to use medication, and to a lesser extent newer types of medication. Quite unexpectedly, people with ID had a lower prevalence of hypertension than the general population, especially when considering only diagnoses and not drug prescriptions. If this is due to failure to diagnose hypertension among people with ID, or differences in types of health care used, is unknown. Treatment regimens for hypertension also differed among people with ID and the general populations such that people with ID more often were prescribed older types of medications. Therefore, it is of great importance to investigate if the people with ID and diabetes mellitus or hypertension are subjected to the same regular drug review that is recommended for older people in general in order to decrease the risk of CVD in this population.

As the present study was register based, we were only able to present data on diagnoses made and medications prescribed. Research in clinical settings is needed to obtain further knowledge about the treatment regimens used among older people with ID, and whether these are in accordance with their health problems. Also, to assess how older people with ID experience their disease and the treatment and health care they receive.

\section{Additional files}

Additional file 1: Number of people with each type of diabetes mellitus and hypertension diagnosis in a cohort of people with intellectual disability (ID) and a random sample from the general population (gPop). Note! Each person may have more than one type of diagnosis. (DOCX $15 \mathrm{~kb}$ )

Additional file 2: Number of people with at least one prescription of each drug used in diabetes mellitus and drugs for hypertension, respectively, during 2006-2012 in a group of people with intellectual disability (ID, $n=7936)$ and a same-sized sample from the general population (gPop). (DOCX $15 \mathrm{~kb}$ )

Additional file 3: Prescription of drugs used for diabetes mellitus among those with at least one such diagnosis in a group of people with intellectual disability (ID) and referents from the general population (gPop). (DOCX $15 \mathrm{~kb}$ )

\section{Abbreviations}

ACE: Angiotensin-Converting-Enzyme; ATC: Anatomical Therapeutic Chemical; Cl: Confidence Interval; CVD: Cardiovascular disease;

GLM: Generalized Linear Model; gPop: General Population; ICD: International Statistical Classification of Diseases and Related Health Problems;

ID: Intellectual Disability; RR: Relative Risk

\section{Acknowledgements}

We would like to acknowledge the cooperation of the FUB (The Swedish National Association for People with Intellectual Disability).

\section{Funding}

This work was funded by Forte, the Swedish Research Council for Health, Working Life and Welfare no. 2014-4753. The funding body had no part in the design of the study, collection, analysis, and interpretation of data, or writing the manuscript.

\section{Availability of data and materials}

The data in the present study contains sensitive information on a very vulnerable group, i.e. people with ID. Even though the data are anonymized, it contains enough details to enable identification of single individuals. Therefore, in order to approve the study, the Regional Ethical Review Board in Lund made considerable restrictions regarding access to the data. This means we will not be able to provide other researchers with our data. However, as our database is compiled by register data only, other researchers may contact Statistics Sweden and the Swedish National Board of Health and Welfare to get access to the different registers included, and thereby recreate the database.

\section{Authors' contributions}

$\mathrm{GA}$, the $\mathrm{Pl}$, initiated the research project regarding aging with intellectual disability in Sweden, and managed the acquisition of resources and data. All authors participated in the design of the present study concerning chronic disorders that are risk factors for cardiovascular disease within this project. AA performed the data management and statistical analyses, and wrote the manuscript. All authors took part in the interpretation of data and consideration of potential implications, as well as revising of the manuscript. All authors read and approved the final manuscript.

\section{Author's information}

AA (PhD, associate professor) is a statistician and epidemiologist. GA (RN, $\mathrm{PhD}$, professor) has long experience in research regarding disability and health care for the elderly. PH (MD, PhD, professor) has expertise in pharmacology as well as in ethics.

\section{Ethics approval and consent to participate}

Approval was obtained from the Regional Ethical Review Board in Lund (no. 2013/15). The National Board of Health and Welfare performed a separate secrecy review in 2014 before providing access to the data. All analyses were performed using anonymized datasets. The authors assert that all procedures contributing to this work comply with the ethical standards of the relevant national and institutional committees on human experimentation and with the Helsinki Declaration of 1975, as revised in 2008.

Data in the present study is based on anonymized information provided by two official national registrars, The National Board of Health and Welfare and Statistics Sweden. These authorities provide anonymized information for research purposes to individual researchers once the study has been vetted and approved by the Regional Ethical Review Board according to Swedish ethical review regulations. Because of the requirement of anonymized data, each individual could not be asked for consent to participate; instead active refusal of participation was applied. This was done by publishing information about the planned study in the national newspaper" Dagens Nyheter" and in UNIK, the magazine of The Swedish National Association for People with Intellectual Disability (FUB), which is printed in 22,000 copies. The target audience for the UNIK magazine are mainly members (people with ID) and their families. The advertisement was written in two versions, whereof one was easy-to-read text. The advertisement presented the study and contained information on how to contact the research manager (GA) by phone, email or mail in order to opt out of the study. The research manager (GA) was then responsible for contacting the national registrars so that those who opted out were excluded before the registrars provided any data to the research manager.

Consent for publication

Not applicable.

Competing interests

The authors declare that they have no competing interests. 


\section{Publisher's Note}

Springer Nature remains neutral with regard to jurisdictional claims in published maps and institutional affiliations.

\section{Author details}

'Division of Occupational and Environmental Medicine, Faculty of Medicine, Lund University, SE-221 00 Lund, Sweden. 'Department of Health Sciences, Faculty of Medicine, Lund University, Lund, Sweden. ${ }^{3}$ Division of Clinical Chemistry and Pharmacology, Faculty of Medicine, Lund University, Lund, Sweden.

Received: 27 March 2017 Accepted: 9 November 2017 Published online: 23 November 2017

\section{References}

1. WHO. Global Atlas on cardiovascular disease Prev Control 2011

2. Li H, Oldenburg B, Chamberlain C, O'Neil A, Xue B, Jolley D, Hall R, Dong Z, Guo Y. Diabetes prevalence and determinants in adults in China mainland from 2000 to 2010: a systematic review. Diabetes Res Clin Pract. 2012;98(2): 226-35

3. Garcia-Dominic O, Lengerich EJ, Camacho F, Gallant NR, Wray LA, Ahern F, Bogdan G, Weinberg G, Ulbrecht JS. Prevalence of diabetes and associated obesity in Pennsylvania adults, 1995-2010. Prev Chronic Dis. 2014;11:E111.

4. Hajjar I, Kotchen JM, Kotchen TA. HYPERTENSION: Trends in prevalence, incidence, and control. Annu Rev Public Health. 2006:27(1):465-90.

5. Haveman M, Perry J, Salvador-Carulla L, Walsh PN, Kerr M, Van Schrojenstein Lantman-de Valk H, Van Hove G, Berger DM, Azema B, Buono S, et al. Ageing and health status in adults with intellectual disabilities: results of the European POMONA II study. J Intellect Develop Disabil. 2011;36(1):49-60.

6. Sohler N, Lubetkin E, Levy J, Soghomonian C, Rimmerman A. Factors associated with obesity and coronary heart disease in people with intellectual disabilities. Soc Work Health Care. 2009:48(1):76-89.

7. de Winter CF, Bastiaanse LP, Hilgenkamp TI, Evenhuis HM, Echteld MA. Cardiovascular risk factors (diabetes, hypertension, hypercholesterolemia and metabolic syndrome) in older people with intellectual disability: results of the HA-ID study. Res Dev Disabil. 2012;33(6):1722-31.

8. van de Louw J, Vorstenbosch R, Vinck L, Penning C, Evenhuis H. Prevalence of hypertension in adults with intellectual disability in the Netherlands. J Intellect Disabil Res. 2009;53(1):78-84.

9. Haveman M, Perry J, Salvador-Carulla L, Walsh PN, Kerr M, Lantman-de Valk HV, Van Hove G, Berger DM, Azema B, Buono S, et al. Ageing and health status in adults with intellectual disabilities: results of the European POMONA II study. J Intellect Develop Disabil. 2011;36(1):49-60.

10. MacRae S, Brown M, Karatzias T, Taggart L, Truesdale-Kennedy M, Walley R, Sierka A, Northway R, Carey M, Davies M. Diabetes in people with intellectual disabilities: a systematic review of the literature. Res Dev Disabil. 2015;47:352-74.

11. Emerson E, Hatton C, Baines S, Robertson J. The physical health of British adults with intellectual disability: cross sectional study. Int J Equity Health. 2016;15:11.

12. Balogh RS, Lake JK, Lin E, Wilton A, Lunsky Y. Disparities in diabetes prevalence and preventable hospitalizations in people with intellectua and developmental disability: a population-based study. Diabet Med. 2015;32(2):235-42

13. Haider SI, Ansari Z, Vaughan L. Matters H, Emerson E. Health and wellbeing of Victorian adults with intellectual disability compared to the general Victorian population. Res Dev Disabil. 2013;34(11):4034-42.

14. McCarthy J, O'Hara J. III-health and intellectual disabilities. Curr Opin Psychiatry. 2011;24(5):382-6.

15. Bailey CJ. The current drug treatment landscape for diabetes and perspectives for the future. Clin Pharmacol Ther. 2015;98(2):170-84.

16. Phung OJ, Sobieraj DM, Engel SS, Rajpathak SN. Early combination therapy for the treatment of type 2 diabetes mellitus: systematic review and metaanalysis. Diabetes Obes Metab. 2014;16(5):410-7.

17. Pfeiffer AF, Klein HH. The treatment of type 2 diabetes. Dtsch Arztebl Int 2014;111(5):69-81. quiz 82

18. Kruger DF, Boucher $\mathrm{J}$, Banerji MA. Utilizing current diagnostic criteria and treatment algorithms for managing type 2 diabetes mellitus. Postgrad Med. 2011;123(4):54-62.
19. Davidson JA. The placement of DPP-4 inhibitors in clinical practice recommendations for the treatment of type 2 diabetes. Endocr Pract. 2013: 19(6):1050-61.

20. Kim KS, Kim SK, Sung KM, Cho YW, Park SW. Management of type 2 diabetes mellitus in older adults. Diabetes Metab J. 2012;36(5):336-44.

21. Kjeldsen SE, Stenehjem A, Os I, Van de Borne P, Burnier M, Narkiewicz K, Redon J, Agabiti Rosei E, Mancia G. Treatment of high blood pressure in elderly and octogenarians: European Society of Hypertension statement on blood pressure targets. Blood Press. 2016;25(6):333-6.

22. Benetos A, Bulpitt CJ, Petrovic M, Ungar A, Agabiti Rosei E, Cherubini A, Redon J, Grodzicki T, Dominiczak A, Strandberg T, et al. An expert opinion from the European Society of Hypertension-European Union Geriatric Medicine Society Working Group on the Management of Hypertension in very old, frail subjects. Hypertension. 2016;67(5):820-5.

23. SFS1993:387: Act Concerning Support and Service for Persons with Certain Functional Impairments (In Swedish: Lag om stöd och service till vissa funktionshindrade (LSS)). In. Stockholm, Sweden; 1993.

24. Wettermark B, Hammar N, Fored CM, Leimanis A, Otterblad Olausson P, Bergman U, Persson I, Sundström A, Westerholm B, Rosén M. The new Swedish prescribed drug register-opportunities for pharmacoepidemiological research and experience from the first six months. Pharmacoepidemiol Drug Saf. 2007;16(7):726-35.

25. Sandberg M, Ahlström G, Axmon A, Kristensson J. Somatic healthcare utilisation patterns among older people with intellectual disability: an 11 year register study. BMC Health Serv Res. 2016;16(1):642.

26. Axmon A, Björne P, Nylander L, Ahlström G. Psychiatric care utilization among older people with intellectual disability in comparison with the general population: a register study. BMC Psychiatry. 2016;16(1):389.

27. Sandberg M, Ahlström G, Kristensson J. Patterns of somatic diagnoses in older people with intellectual disability: a Swedish eleven year case-control study of inpatient data. J Appl Res In Intellect dis: JARID. 2017:30(1):157-71.

28. Socialstyrelsen. Diabetes care - efforts of the county councils and municipalities (In Swedish: Diabetesvård - landstingens och kommunernas insatser). 2012.

29. Boles A, Kandimalla R, Reddy PH. Dynamics of diabetes and obesity: epidemiological perspective. Biochim Biophys Acta. 2017; 1863(5):1026-36.

30. Robertson J, Emerson E, Baines S, Hatton C. Obesity and health behaviours of British adults with self-reported intellectual impairments: cross sectional survey. BMC Public Health. 2014;14:219.

31. Wilson PW, D'Agostino RB, Sullivan L, Parise H, Kannel WB. Overweight and obesity as determinants of cardiovascular risk: the Framingham experience. Arch Intern Med. 2002;162(16):1867-72.

32. Pan $A$, Wang $Y$, Talaei $M, F B$ H, Relation $W T$. Of active, passive, and quitting smoking with incident type 2 diabetes: a systematic review and metaanalysis. The lancet Diabetes \& endocrinology. 2015;3(12):958-67.

33. Virdis A, Giannarelli C, Neves MF, Taddei S, Ghiadoni L. Cigarette smoking and hypertension. Curr Pharm Des. 2010:16(23):2518-25.

34. Beilin LJ, Puddey IB. Alcohol, hypertension and cardiovascular diseaseimplications for management. Clin Exp Hypertens. 1993;15(6):1157-70.

35. Knott C, Bell S, Britton A. Alcohol consumption and the risk of type 2 diabetes: a systematic review and dose-response meta-analysis of more than 1.9 million individuals from 38 observational studies. Diabetes Care. 2015;38(9):1804-12.

36. Melville CA, Hamilton S, Hankey CR, Miller S, Boyle S. The prevalence and determinants of obesity in adults with intellectual disabilities. Obes Rev. 2007:8(3):223-30.

37. Läkemedelsverket. Drug therapy for type 2 diabetes - new recommendations (In Swedish: Läkemedelsbehandling vid typ 2-diabetes ny rekommendation). 2010.

38. Evenhuis HM, Hermans H, Hilgenkamp TI, Bastiaanse LP, Echteld MA Frailty and disability in older adults with intellectual disabilities: results from the healthy ageing and intellectual disability study. J Am Geriatr Soc. 2012;60(5):934-8

39. Razzaghi $\mathrm{H}$, Oster M, Reefhuis J. Long-term outcomes in children with congenital heart disease: National Health Interview Survey. J Pediatr. 2015; 166(1):119-24

40. D'Alto M, Mahadevan VS. Pulmonary arterial hypertension associated with congenital heart disease. Eur Respir Rev. 2012;21(126):328-37.

41. Susic D, Varagic J. Obesity: a perspective from hypertension. Med Clin North Am. 2017;101(1):139-57. 